\title{
Sliding mode based robustification of consensus and distributed optimization control protocols
}

\author{
Alessandro Pilloni, Mauro Franceschelli, Alessandro Pisano, Elio Usai
}

\begin{abstract}
This paper proposes a design approach, based on the integral sliding mode control paradigm, devoted to give robustness to multi-agent systems executing arbitrary distributed optimization and consensus protocols which do not take this feature into account. Robustness is understood as the capability of rejecting the effect of exogenous disturbances, parameter uncertainties, and uncertain couplings between the agents dynamics, by achieving the same emerging behaviour as that corresponding to a reference MAS designed to achieve a given coordination objective in the nominal case. The proposed approach yields a distributed state feedback which can seamlessly be integrated into existing distributed optimization and cooperative control protocols which are usually prone to disturbances and uncertainties corrupting the MAS dynamics. Nonsmooth Lyapunov analysis supports the claimed properties. Numerical simulations, showing how popular distributed optimization and consensus protocols can effectively be robustified are discussed.
\end{abstract}

Index Terms-Multi-agent systems, Sliding mode control, Distributed control, Robust control, Consensus, Nonsmooth analysis.

\section{INTRODUCTION}

D istributed control of Multi-Agent Systems (MASs) finds application in many relevant domains such as smart grids control [1], [2], sensor networks [3], multi-robot systems [4], [5], distributed optimization [6], [7].

In particular, intense research efforts have been spent for the so-called "leaderless consensus" problem, namely the problem of designing local interaction rules such that the agents approach/estimate, in a cooperative way, a common unknown a-priory quantity consisting on the solution of a welldefined coordination task. For instance, the mean of agent's initial conditions as in the average consensus problem.

Most of the existing consensus algorithms are designed by exploiting the same tools of distributed optimization, for instance, the sub-gradient descent method, [6], [8], [9], [10]. Thus, they can be treated as instances of a distributed optimization.

However, since leader-less coordination tasks require adhoc equilibrium conditions on the agents' dynamics, their convergence and stability properties are not preserved when uncertainties corrupt their local dynamics [1]-[10].

The research leading to these results has received funding by: Fondazione di Sardegna under projects "ODIS" (CUP:F72F16003170002) and "SISCO" (CUP:F74I19001060007); by the Italian Ministry of Research and Education (MIUR) by project "CoNetDomeSys" (code:RBSI14OF6H) under call SIR 2014; by the Sardinian Regional Government, by project "Virtual Energy" under call "Cluster top-down actions (POR FESR)", and project MOSIMA (RASSR05871), FSC 2014-2020, annuity 2017, Subject area 3, Action Line 3.1., and POR SARDEGNA FSE 2014-2020-Asse III, Azione 10.5.12, "Avviso di chiamata per il finanziamento di Progetti di ricerca Anno 2017”.

A. Pilloni, M. Franceschelli, A. Pisano, E. Usai are with the Department of Electrica and Electronic Engineering (DIEE), University of Cagliari, Cagliari, 09123, Italy. Corresponding author: Mauro Franceschelli, email: mauro.franceschelli@unica.it.
To face off robustness issues, and whenever agents consist of physical systems affected by model mismatching or perturbations, ad-hoc designs need to be carried out [11]-[22].

In the literature, the robustness issue has been addressed from different perspectives. In [11], the consensus problem in the presence of measurements errors is addressed by means of linear consensus protocols with time-varying weights. In [12] the so-called " $\varepsilon$-consensus" is introduced, mostly characterizing the effects of perturbations rather than attempting their rejection or attenuation.

In [13], the finite-time synchronization problem is solved for networks of perturbed single integrators affected by unknown bounded couplings and disturbances. Moreover, in [14] under certain restrictions on the directed switching communication topology which was allowed to not be always connected, and perturbations, it is shown that agents perform $\varepsilon$-consensus.

Robust control techniques such as Sliding Mode Control [13], [14], [15], [16], Linear Matrix Inequality-based schemes [17], Lipschitz finite-time controllers [18], and Internal Model Control [19], [20] have also been exploited for cooperative control applications. However, the complete rejection of perturbations has been achieved only for specific problems. For instance, the distributed tracking by the follower agents of a leader agent [1], [21], [22], or the agents synchronization towards common quantities depending also by the perturbations, see applications on clocks [13], [14], [18], thermal [15] and Lagrangian systems [16].

Apart from the mentioned problems, only strategies capable of guaranteeing a performance trade-off between robustness and accuracy are available, see [11], [12], [17], [19], [20].

Main contribution: This paper presents a robust protocol re-design process targeted to provide robustness in uncertain MASs executing arbitrary distributed optimization and consensus protocols which do not take robustness into account.

Robustness is understood as the capability of rejecting disturbances, parameters uncertainties, and uncertain physical couplings between agents. The contribution of the present paper is relevant to distributed control and optimization protocols which do not consider uncertain agents' dynamics.

Under the standard assumption that an upper bound, possibly either state- or time-dependent, to the magnitude of the uncertainties is available [1], [13]-[18], we show how to design a robustified local interaction protocol to force a desired emergent behaviour, expected for an ideal multi-agent system of unperturbed $d$-dimensional integrators under a given "reference" local interaction rule, to a multi-agent system with $d$-dimensional first-order agents with nonlinear uncertain and coupled dynamics.

In contrast with the few algorithms enabling the perturba- 
tions rejection, see for instance [13]-[18], with the method proposed in this paper the consensus value is not influenced by either uncertainties or perturbations. Thus, our proposal is well-suited also to gradient-based distributed optimization tasks, which may be affected by uncertainties and perturbations corrupting the gradient descent direction provided by the control terms. Our contribution is, thus, a general-purpose redesign process rather than an ad-hoc design for a specific application.

The design is based on a distributed implementation of the Integral Sliding Mode Control (ISMC) paradigm [23]. Close to the spirit of this study, in [24] the ISMC was used to robustify centralized LQ controllers. Attempts to integrate the ISMC approach within MASs were made in [21] and [22] for leader-follower applications. Despite ISMC assumes full state availability, its design is here reformulated in a way that the communication constraints are met, and each local controller only needs its own state information and that of its neighbors. This approach can be interpreted as a method to force an uncertain networked system to track the emerging behaviour exhibited by a reference MAS tasked to solve a given coordination problem, either consensus or distributed optimization task.

Since the resulting control protocol is discontinuous, Filippov theory [25] and nonsmooth Lyapunov analysis support the treatment [26], [27]. Preliminary results, limited to the average consensus problem and without involving nonsmooth analysis concepts were presented in [28].

Paper organization: In Section II the adopted notation and basic notions on nonsmooth theory are summarized. The problem formulation and the proposed solution are described in Section III along with the stability analysis. A suitable smooth reformulation of the proposed protocol devoted to alleviate chattering in practical implementation is discussed in Section IV. Numerical case studies are discussed in Section V. Finally, in Section VI, some concluding remarks are given.

\section{Mathematical Preliminaries and Notations}

A. Notation: The sets of natural and strictly positive real numbers are denoted by $\mathbb{N}$ and $\mathbb{R}_{\geqslant 0}$. For $d \in \mathbb{N}$ and a column vector $x=\left(x_{1}, \ldots, x_{d}\right)^{\top} \in \mathbb{R}^{d}, x_{i} \in \mathbb{R}$, let $x^{\top}$ be its transpose, and let $\|x\|_{1}=\sum_{i=1}^{d}\left|x_{i}\right|,\|x\|_{\infty}=\max _{i}\left\{x_{i}\right\}$ be the 1 - and $\infty$-norms of $x$. Let $\mathbf{1}=(1, \ldots, 1)^{\top} \in \mathbb{R}^{p}$ and $\mathbf{0}=(0, \ldots, 0)^{\top} \in \mathbb{R}^{p}$ whereas $\boldsymbol{I}_{p} \in \mathbb{R}^{p \times p}$ is the identity matrix. For $x$ and $y$, let $x^{\top} \cdot y$ and $x \otimes y$ be the scalar and the Kronecker product. $x^{\top} \cdot y \leqslant\left|x^{\top} \cdot y\right| \leqslant\|x\|$. $\|y\|$. The gradient of a function $V(x): \mathbb{R}^{d} \mapsto \mathbb{R}$ with respect to its argument is denoted by $\nabla V$. Let $\mathscr{S}$ be a set of points in $\mathbb{R}^{p}, \operatorname{co}\{\mathscr{S}\}$ and $\overline{c o}\{\mathscr{S}\}$ denote the convex hull and the convex closure of $\mathscr{S}$. Let $\operatorname{diag}\left(\left(\mathbb{R}^{p}\right)^{n}\right)=\left\{(x, \ldots, x) \in\left(\mathbb{R}^{p}\right)^{n} \mid x \in \mathbb{R}^{p}\right\}$, for $n \in \mathbb{N}$. We denote by $\operatorname{sign}(\cdot): \mathbb{R}^{d} \rightarrow \mathbb{R}^{d}$ the operator which outputs a vector whose entry is 1 if the sign of the corresponding input vector element is positive, -1 if it is negative, 0 otherwise. $\mathrm{V}=\{1, \ldots, n\}$ denotes the set of $n$ agents operating over a communication network whose topology is encoded by a directed graph $G(V, E) . E \subseteq\{V \times V\}$ is the set of edges representing the communication links with real weights $a_{i j}>$ $0 \Leftrightarrow(i, j) \in \mathrm{E}, a_{i j}=0$ otherwise. The "closed neighborhood" of agent $i$ is denoted by $\mathrm{N}_{i}=\{i\} \cup\{j \in \mathrm{V}:(i, j) \in \mathrm{E}\}$. The vector collecting the states of all the agents within $\mathrm{N}_{i}$ is denoted by $x_{j \in \mathrm{N}_{i}}$.

B. Filippov solutions: Consider the differential equation

$$
\dot{x}(t)=\boldsymbol{g}(x, t)
$$

where $\boldsymbol{g}: \mathbb{R}^{p} \times \mathbb{R} \rightarrow \mathbb{R}^{p}$ is measurable and essentially locally bounded, i.e., $\exists \boldsymbol{G}(x, t):\|\boldsymbol{g}(x, t)\| \leqslant \boldsymbol{G}(x, t)$ except on sets of Lebesgue measure zero $\mathscr{N}$ in $\mathbb{R}^{p}$.

Definition 1 ([25]): A vector function $x(t) \in \mathbb{R}^{p}$ is a "Filippov solution" of (1) on $\mathscr{T}:=\left[t_{0}, t_{1}\right]$, if $x(t)$ is absolutely continuous on $\mathscr{T}$ and for almost all $t \in \mathscr{T}$ satisfies the differential inclusion

$$
\dot{x}(t) \in \mathrm{K}[\boldsymbol{g}(x, t)] \equiv \bigcap_{r>0} \bigcap_{\mu(\mathscr{N})=0} \overline{c o} \boldsymbol{g}(B(x, r)-\mathscr{N}, t),
$$

where $B(x, r)$ is an open ball, centered at $x=x(t)$, of radius $r>0$ and $\cap_{\mu(\mathscr{N})=0}$ denotes the intersection over all sets $\mathscr{N}$ of Lebesgue measure zero in $\mathbb{R}^{p}$.

Since $\mathrm{K}[\boldsymbol{g}(x, t)]: \mathbb{R}^{p} \times \mathbb{R} \rightarrow 2^{\mathbb{R}^{p}}$ is locally bounded, upper semi-continuous with nonempty compact convex values, the Filippov solutions' existence is guaranteed [25]. Details on how to derive differential inclusions can be found in [26].

C. Tools for nonsmooth stability analysis: From Rademacher's Theorem, locally Lipschitz functions are differentiable almost everywhere (a.e.). Next, we recall the definition of Clarke's generalized gradient of a locally Lipschitz function.

Definition 2 (Clarke's generalized gradient [29]): Let $V(x): \mathbb{R}^{p} \rightarrow \mathbb{R}$ be Lipschitz near $x \in \mathbb{R}^{p}, \mathscr{N}$ be any set of zero measure in $\mathbb{R}^{p}$, and $W_{V} \subset \mathbb{R}^{p}$ is the set of points where $V$ fails to be differentiable, the Clarke's generalized gradient of $V$ is $\partial V(x) \equiv \operatorname{co}\left\{\lim \nabla V\left(x_{i}\right): x_{i} \rightarrow x, x_{i} \notin W_{V} \cup \mathscr{N}\right\}$.

The set-valued map $\partial V$ consists of all the convex combinations between all of the possible limits of $\nabla V$ at neighboring points of $x$ where $V$ is differentiable. A function $V(x)$ is regular at $x$ provided that it is Lipschitz near $x$, and admits directional derivatives at $x$ for all directions. Continuously differentiable functions at $x$ are also regular at $x$. Also, convex functions which are Lipschitz near $x$ are regular at $x$ (cf. [30, Chapter 2]). Next, we recall a fundamental property of Filippov solutions.

Theorem 1 (Chain rule [27]): Let $x(t)$ be a Filippov solution to (2) on an interval containing $t$ and $V: \mathbb{R}^{p} \times \mathbb{R} \rightarrow \mathbb{R}$ be a Lipschitz and regular function. Then $V(x(t), t)$ is absolutely continuous, $(d / d t)[V(x(t), t)]$ exists a.e. and

$$
\frac{d}{d t}[V(x(t), t)] \epsilon^{\text {a.e. }} \dot{\tilde{V}}(x, t):=\bigcap_{\boldsymbol{\zeta} \in \partial V(x, t)} \boldsymbol{\zeta}^{\top}\left(\begin{array}{c}
\mathrm{K}[\boldsymbol{g}(x, t)] \\
1
\end{array}\right) .
$$

Theorem 2 (Generalized Lyapunov Theorem [26]): If 1) $V(x): \mathbb{R}^{p} \rightarrow \mathbb{R}, V(0)=0$, and $V(x)>0 \forall x \neq 0$, and 2) $x(t): \mathbb{R} \rightarrow \mathbb{R}^{p}$ and $V(x(t))$ is absolutely continuous on $\left[t_{0}, \infty\right)$ with generalized time derivative

$$
\frac{d}{d t}[V(x(t))]<-\varepsilon<0 \quad \text { a.e. on } \quad\{t \mid x(t) \neq 0\}
$$

then $x$ converges to zero in finite-time. 


\section{Problem Formulation and Main Results}

Consider MAS composed of $n$ agents enabled to exchange information with each other through a communication network whose topology is encoded by a directed time-invariant graph $\mathrm{G}(\mathrm{V}, \mathrm{E})$. The agents' dynamics are described by

$$
\dot{x}_{i}(t)=m_{i}\left(x_{j \in \mathrm{N}_{i}}, t\right)+u_{i}(t), \quad x_{i}(0) \in \mathbb{R}^{d}, \quad i \in \mathrm{V},
$$

where $x_{i}=\left(x_{i, 1}, \ldots, x_{i, d}\right)^{\top}$ is the state of the agent $i$ and $u_{i} \in \mathbb{R}^{d}$ is the local control vector. The nonlinear term $m_{i}\left(x_{j \in \mathrm{N}_{i}}, t\right)$ denotes the uncertain agent's dynamics which are allowed to depend on the state of agents in the closed neighborhood of agent $i$ so as to account for possible uncertain couplings between agents, perturbations and parameter uncertainties.

Model (4) may represent generators connected to the power system [1], [2], [31], coupled oscillators [31], [32], mobile robots [4], [5], or else. The uncertain term $m_{i}(\cdot, t)$ is not required to be smooth but simply essentially locally bounded.

Assumption 1: Let $\bar{m}_{i}(\cdot, t): \mathbb{R}^{d} \times, \ldots, \times \mathbb{R}^{d} \times \mathbb{R} \rightarrow \mathbb{R}^{d}$ be a nominal model of $m_{i}(\cdot, t)$ in (4), and let $\tilde{m}_{i}(\cdot, t)=m_{i}(\cdot, t)-$ $\bar{m}_{i}(\cdot, t)$ be the model mismatch. We assume $\tilde{m}_{i}(\cdot, t)$ to be unknown and essentially locally bounded. Particularly, there exists a known state- and time-dependent scalar upper bound function $\delta_{i}(\cdot, t)$, called "safety margin", such that

$$
\left\|\tilde{m}_{i}\left(x_{j \in \mathrm{N}_{i}}, t\right)\right\|_{\infty}<\text { a.e. } \delta_{i}\left(x_{j \in \mathrm{N}_{i}}, t\right) .
$$

Remark 1: Whenever an internal model characterization of the uncertainties is not available the knowledge of a safety margin is widely accepted, and ubiquitous in the sliding-mode control area [1], [8], [13]-[16], [18]-[22], [23]-[26], [33], [34]-[37]. It can be derived on the basis of the uncertainties structure, or by means of "worst case" considerations. It would be small, if the nominal part of $m_{i}(\cdot, t)$ is known with a sufficient degree of accuracy. Otherwise, it would be large thus yielding large, and possibly unnecessary, control effort. This drawback can be alleviated if adaptive (or "anti-chattering") versions of sliding mode control are adopted [35], [36]. In Section IV, a similar reformulation for the proposed control strategy, that sacrifices the ideal accuracy to counteract the control authority thereby limiting the effects of a large-inmagnitude discontinuous control, is provided.

Preliminary considerations: Most of the works in the literature address consensus, distributed control and distributed optimization problems by the design of local interaction rules $f_{i}(\cdot, t): \mathbb{R}^{d} \times, \ldots, \times \mathbb{R}^{d} \times \mathbb{R} \rightarrow \mathbb{R}^{d}$ between agents modeled as ideal continuous-time integrators unaffected by uncertainties, i.e.

$$
\dot{\hat{x}}_{i}(t)=f_{i}\left(\hat{x}_{j \in \mathrm{N}_{i}}, t\right), \quad \hat{x}_{i}(0) \in \mathbb{R}^{d}, \quad i \in \mathrm{V} .
$$

where $f_{i}(\cdot, t)$ abides the topological constraints of the communication links represented by a given graph $G(V, E)$. Without loss of generality, since it represents a feasible coordination protocol, $f_{i}(\cdot, t)$ is assumed essentially locally bounded.

Problem formulation: Instead of developing and proving the convergence properties of an ad-hoc control protocol robust to perturbations, we aim to develop a general methodology which can be applied to any existing distributed local interaction protocol $f_{i}(\cdot, t)$ and implemented by perturbed agents modeled as in (4) instead of ideal $d$-dimensional integrators as in (5).

In particular, let $\hat{x}_{i}(0)=x_{i}(0)$ in (5), where $x_{i}(0)$ is the initial state of the agents (4), we design a control input $u_{i}(t)$ for (4) such that the state trajectories of the agents (4) match the "nominal" solutions of (5) since the initial time instant, i.e.,

$$
x_{i}(t)=\hat{x}_{i}(t), \quad \forall t \in \mathbb{R}_{\geqslant 0}, \quad \forall i \in \mathrm{V} .
$$

Main results: Next, we provide the main results.

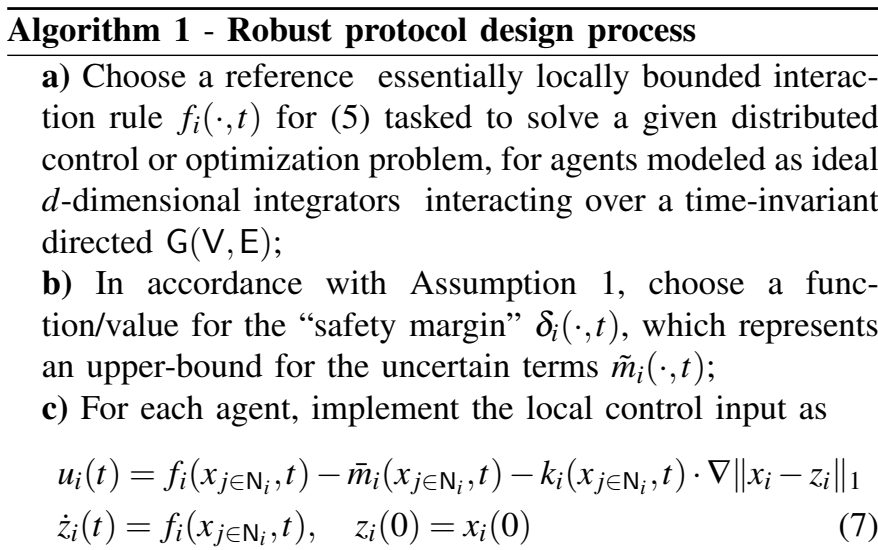

where $z_{i} \in \mathbb{R}^{d}$ is an auxiliary control variable, $k_{i}(\cdot, t): \mathbb{R}^{d} \times$ $\cdots \times \mathbb{R}^{d} \times \mathbb{R} \rightarrow \mathbb{R}$ is a function of class $\mathscr{C}^{0}$;

d) If the local control gain $k_{i}(\cdot, t)$, for each agent, bounds from above the safety margin $\delta_{i}(\cdot, t)$, according to Theorem 3 the state trajectories of the agents (4) track exactly those of the desired reference local interaction rule (5).

Theorem 3: Consider the uncertain MAS dynamics (4) with the local control (7). Let Assumption 1 be in force, and $\hat{x}_{i}(0)=$ $x_{i}(0)$ in (5). If $k_{i}(\cdot, t)>\delta_{i}(\cdot, t)$ then relation (6) is in force.

Proof: We first substitute (7) into (4) to obtain a compact representation of the collective network dynamics

$$
\begin{aligned}
& \dot{\boldsymbol{x}}(t)=\tilde{\boldsymbol{m}}(\boldsymbol{x}, t)+\boldsymbol{f}(\boldsymbol{x}, t)-\boldsymbol{k}(\boldsymbol{x}, t) \nabla\|\boldsymbol{x}-\boldsymbol{z}\|_{1} \\
& \dot{\boldsymbol{z}}(t)=\boldsymbol{f}(\boldsymbol{x}, t), \quad \boldsymbol{z}(0)=\boldsymbol{x}(0)
\end{aligned}
$$

where $\boldsymbol{x}=\left(x_{1}^{\top}, \ldots, x_{n}^{\top}\right)^{\top}, \quad \boldsymbol{z}=\left(z_{1}^{\top}, \ldots, z_{n}^{\top}\right)^{\top}, \quad$ and $\boldsymbol{f}=$ $\left(f_{1}^{\top}, \ldots, f_{n}^{\top}\right)^{\top}$ are column vectors in $\left(\mathbb{R}^{d}\right)^{n}$, whereas

$$
\begin{aligned}
& \boldsymbol{k}(\boldsymbol{x}, t)=\operatorname{diag}(\overbrace{k_{1}\left(x_{j \in \mathrm{N}_{1}}, t\right) \otimes \boldsymbol{I}_{d}}^{\boldsymbol{k}_{1}(\cdot, t)}, \ldots, \overbrace{k_{n}\left(x_{j \in \mathrm{N}_{n}}, t\right) \otimes \boldsymbol{I}_{d}}^{\boldsymbol{k}_{n}(\cdot, t)}) \\
& \tilde{\boldsymbol{m}}(\boldsymbol{x}, t)=\left(\tilde{m}_{1}\left(x_{j \in \mathrm{N}_{1}}, t\right)^{\top}, \ldots, \tilde{m}_{n}\left(x_{j \in \mathrm{N}_{n}}, t\right)^{\top}\right)^{\top}
\end{aligned}
$$

Let us define the argument of the nonsmooth term in (8) as

$$
\boldsymbol{\sigma}(t)=\boldsymbol{x}(t)-\boldsymbol{z}(t), \quad \boldsymbol{\sigma} \in\left(\mathbb{R}^{d}\right)^{n} .
$$

The subset of elements of $\boldsymbol{\sigma}=\left(\boldsymbol{\sigma}_{1}^{\top}, \ldots, \boldsymbol{\sigma}_{n}^{\top}\right)^{\top}$ related to agent $i$ is denoted as $\sigma_{i} \in \mathbb{R}^{d}$, and the $\ell$-th entry of $\sigma_{i}$ is denoted as

$$
\boldsymbol{\sigma}_{i, \ell}(t)=x_{i, \ell}(t)-z_{i, \ell}(t), \quad i \in \mathrm{V}, \ell=1,2, \ldots, d .
$$


We now rewrite (8), (9) in the new $(\boldsymbol{x}, \boldsymbol{\sigma})$ coordinates as

$$
\begin{aligned}
& \dot{\boldsymbol{x}}(t)=\tilde{\boldsymbol{m}}(\boldsymbol{x}, t)+\boldsymbol{f}(\boldsymbol{x}, t)-\boldsymbol{k}(\boldsymbol{x}, t) \cdot \nabla\|\boldsymbol{\sigma}(t)\|_{1} \\
& \dot{\boldsymbol{\sigma}}(t)=\tilde{\boldsymbol{m}}(\boldsymbol{x}, t)-\boldsymbol{k}(\boldsymbol{x}, t) \cdot \nabla\|\boldsymbol{\sigma}(t)\|_{1} .
\end{aligned}
$$

Note that, whenever an entry $\boldsymbol{\sigma}_{i, \ell}$ of $\boldsymbol{\sigma}(t)$ equals zero for some $t$, the corresponding entry of the $\nabla\|\boldsymbol{\sigma}(t)\|_{1}$ is not defined in traditional sense. To draw conclusions on the stability of (13)-(14), their solutions must be understood in the Filippov sense. Due to Assumption 1, the right hand side of (13)(14) is essentially locally bounded. Thus, from Definition 1, they admit a Filippov solution [25]. The differential inclusion associated with (14) is as follows

$$
\dot{\boldsymbol{\sigma}}(t) \in^{\text {a.e. }} \mathrm{K}\left[\tilde{\boldsymbol{m}}(\boldsymbol{x}, t)-\boldsymbol{k}(\boldsymbol{x}, t) \cdot \nabla\|\boldsymbol{\sigma}(t)\|_{1}\right] .
$$

Let us now choose as Lyapunov functional candidate the nonsmooth, locally Lipschitz, regular function

$$
V(\boldsymbol{\sigma}(t))=\|\boldsymbol{\sigma}(t)\|_{1}=\sum_{i \in \mathrm{V}} \sum_{\ell=1}^{d}\left|\sigma_{i, \ell}(t)\right|
$$

which, due to that fact that $\boldsymbol{\sigma}(0)=\mathbf{0}$, it satisfies the condition $V(\boldsymbol{\sigma}(0))=0$, cf. (9) and (11). According to Definition 1, the solution $\sigma(t)$ of (15) is absolutely continuous on a compact interval. This in turn implies the absolute continuity of $V(\boldsymbol{\sigma})$.

Let $\partial V$ be the Clarke's generalized gradient of (16). Since the entries $k_{i}(\cdot, t)$ of (10) are $\mathscr{C}^{0}$, then from Properties 2), 5), 6 ), and 7) of [26, Theorem 1], the generalized time derivative of $V$ along (15) meets (3), and satisfies

$$
\begin{aligned}
\frac{d}{d t}[V(\boldsymbol{\sigma})] \quad & \in^{\text {a.e. }} \\
& \bigcap_{\boldsymbol{\zeta} \in \partial V} \boldsymbol{\zeta}^{\top} \cdot \mathbf{K}\left[\tilde{\boldsymbol{m}}(\boldsymbol{x}, t)-\boldsymbol{k}(\boldsymbol{x}, t) \cdot \nabla\|\boldsymbol{\sigma}(t)\|_{1}\right] \\
& \subset \bigcap_{\boldsymbol{\zeta} \in \partial V} \boldsymbol{\zeta}^{\top} \cdot(\mathrm{K}[\tilde{\boldsymbol{m}}(\boldsymbol{x}, t)]-\boldsymbol{k}(\boldsymbol{x}, t) \cdot \partial V(\boldsymbol{\sigma}(t))) \\
& \bigcap_{\boldsymbol{\zeta} \in \partial V} \boldsymbol{\zeta}^{\top} \cdot(\boldsymbol{p}-\boldsymbol{k}(\boldsymbol{x}, t) \cdot \boldsymbol{\beta})
\end{aligned}
$$

which holds, analogously with [26, equation (28)], a.e. for all $\boldsymbol{\zeta} \in \partial V$, some $\beta \in \partial V$, and some $\boldsymbol{p} \in \mathbf{K}[\tilde{\boldsymbol{m}}(\boldsymbol{x}, t)]$. Moreover, let us note that (17) holds for all $\zeta \in \partial V$, thus we can choose

$$
\boldsymbol{\zeta}=\operatorname{argmin}\left\{\|\boldsymbol{\zeta}\|^{2}: \boldsymbol{\zeta} \in \partial V\right\} .
$$

In accordance with the notation in (10)-(12), let us first rewrite $\boldsymbol{\zeta}=\left(\boldsymbol{\zeta}_{1}^{\top}, \ldots, \boldsymbol{\zeta}_{n}^{\top}\right)^{\top}, \boldsymbol{p}=\left(\boldsymbol{p}_{1}^{\top}, \ldots, \boldsymbol{p}_{n}^{\top}\right)^{\top}$, where $\boldsymbol{\zeta}_{i, \ell}$ and $p_{i, \ell}$ be the $\ell$-th entry of, resp., $\zeta_{i}$ and $\boldsymbol{p}_{i}$. Let $k_{i, \ell}(\cdot, t)=k_{i}(\cdot, t)$ be a diagonal entry for the local gain matrix $\boldsymbol{k}_{i}(\cdot, t)$ in (10) satisfying $k_{i}(\cdot, t)-\delta_{i}(\cdot, t) \geqslant \varepsilon_{i}$, for some $\varepsilon_{i}>0$. From (18) and by substituting (10) into (17), it yields that

$$
\begin{aligned}
\frac{d}{d t}[V(\boldsymbol{\sigma})] & \leqslant \bigcap_{\boldsymbol{\zeta} \in \partial V} \sum_{i}\left(\left|\boldsymbol{\zeta}_{i}^{\top} \cdot \boldsymbol{p}_{i}\right|-k_{i} \cdot\left\|\boldsymbol{\zeta}_{i}\right\|^{2}\right) \\
& \leqslant \bigcap_{\boldsymbol{\zeta} \in \partial V} \sum_{i, \ell}\left(\left|\boldsymbol{\zeta}_{i, \ell}\right| \cdot\left|p_{i, \ell}\right|-k_{i} \cdot\left|\boldsymbol{\zeta}_{i, \ell}\right|^{2}\right) \\
& \leqslant-\bigcap_{\boldsymbol{\zeta} \in \partial V} \sum_{i, \ell}\left(\varepsilon_{i} \cdot\left|\boldsymbol{\zeta}_{i, \ell}\right|^{2}+\delta_{i} \cdot\left(\left|\zeta_{i, \ell}\right|^{2}-\left|\zeta_{i, \ell}\right|\right)\right) .
\end{aligned}
$$

Using the calculus for generalized gradients it holds $\partial V(\mathbf{0})=\left([-1,1]^{d}\right)^{n}$, which is the unit cube in $\left(\mathbb{R}^{d}\right)^{n}$. Moreover, due to (7), $\partial V(\sigma(0))=\left([-1,1]^{d}\right)^{n}$. By letting $\sigma_{i, \ell}$ be as in (12), we further observe that if $\sigma_{i, \ell}=0$, then the element $\partial V_{i, \ell}$ of $\partial V$ is multi-valued and belongs to the convex set $[-1,1]$, where $\partial V_{i, \ell} \equiv \partial\left|\sigma_{i, \ell}\right|$. On the other hand, $\partial V_{i, \ell}=\{+1\}$ if $\sigma_{i, \ell}>0$, or $\partial V_{i, \ell}=\{-1\}$ if $\sigma_{i, \ell}<0$. Due to the convexity of $V$, and by [29, Proposition 2.2.9], for $\sigma_{i, \ell} \neq 0$, it results that $\partial V_{i, \ell} \cap(-1,1)=\varnothing$. Thus,

$$
\arg \min _{\zeta_{i, \ell} \in \partial V_{i, \ell}}\left|\zeta_{i, \ell}(t)\right|=1, \quad \forall \sigma_{i} \neq \mathbf{0},
$$

and, from (19) and (3), it follows that $(d / d t)[V(x(t), t)] \leqslant$ $-\min _{i \in \mathrm{V}}\left\{\varepsilon_{i}\right\}$ on $t \in[0, \infty)$. By invoking Theorem 2 , and thanks to (9) and (11), we conclude that: a) the dynamics of (14) are finite-time stable; b) $\sigma=0$ is an invariant set for $t \geq 0$. Moreover, since (16) is radially unbounded, $\boldsymbol{\sigma}=\mathbf{0}$ is a globally finite-time stable equilibrium for (4), (7). In particular, for all intervals of time $t$ of measure zero for which one or more entries $\sigma_{i, \ell}(t)$ of (11) are zero, according to [25], they can be disregarded. Whereas, if one or more $\sigma_{i, \ell}(t)=0$ are zero on a set $\mathscr{M}$ of time values $t$ of positive measure, since Filippov solutions are absolutely continuous, and $\boldsymbol{\sigma}(t)$ is a Filippov solution, then in the same positive time intervals the derivative of the corresponding Filippov solution for those elements which are zero is zero as well, i.e. $\dot{\sigma}_{i, \ell}(t)=0$ a.e. on $t \in \mathscr{M}$. Thus, if $\boldsymbol{\sigma}(t)=\mathbf{0}$ a.e. on $t \in \mathscr{M}$, then $\boldsymbol{\sigma}(t) \in^{\text {a.e. }}\{\mathbf{0}\}, t \in \mathscr{M}$. Since $\boldsymbol{\sigma}=\boldsymbol{x}-\boldsymbol{z}=0$ is an invariant set, then $\boldsymbol{x}(t) \equiv \boldsymbol{z}(t)$ $\forall t \in \mathbb{R}_{\geq 0}$. Thus, the solution $\boldsymbol{x}(t)$ of (4), (7), is driven by the right-hand side of (9), which is in turn equivalent to the nominal reference behaviour (5), thus yielding (6).

The convergence properties of (8)-(9) are independent from the given task in (5). Thus, Algorithm 1 can be seamlessly implemented on agents with dynamics affected by uncertainties and unknown perturbations.

Remark 2: This research is focused on agents modeled by relative degree one uncertain dynamics as in (4), thus our results are applicable to the vast literature on first-order continuous-time agents related to consensus and distributed optimization problems. First-order systems are important in the context of multi-agent systems because by approximating the agents' dynamics it is possible to address formally the behavior of large scale complex networks. Our proposed robustifying control protocol is thus a general approach to add robustness to uncertainties to all distributed control protocols designed in absence of uncertain dynamics for agents as in (4).

\section{Chattering Alleviation}

Due to the finite switching frequency of a real sliding mode control implementation, high-frequency chattering may arise on the local agents dynamics when implementing (7). In digital realization, such a switching frequency is at most equal to the inverse of the sampling-time $T_{s}$. Besides this, due to the worst-case estimation of $\delta_{i}(\cdot, t)$, the magnitude of $k_{i}(\cdot, t)$ can be larger then necessary. To alleviate the chattering, common practice is to smooth-out the "sign" functions by means of some continuous sigmoid or saturation approximating functions [34]. Another approach entails the replacement of the discontinuous term with an estimation of the so-called "equivalent control", obtained as the output of a low pass filter fed by the discontinuous signal [37, Chapter 2.2 and 2.4]. 
Chattering can also be alleviated by resorting to the highorder sliding mode control algorithms [33], by means of which one can transfer the discontinuity on the higher-order time derivatives of the actual plant control at the price, however, at the price of an increase in the information demand about the state of the controlled plant. In the present paper we refer to a chattering alleviation solution originally proposed in [23]. The resulting algorithm, specialized to the present scenario, is

$$
\begin{aligned}
& u_{i}=-\bar{m}_{i}\left(x_{j \in \mathrm{N}_{i}}, t\right)+f_{i}\left(x_{j \in \mathrm{N}_{i}}, t\right)-k_{i}\left(x_{j \in \mathrm{N}_{i}}, t\right) \cdot w_{i} \\
& \tau_{i} \cdot \dot{w}_{i}+w_{i}=\nabla\left\|x_{i}-z_{i}\right\|_{1} \\
& \dot{z}_{i}=f_{i}\left(x_{j \in \mathrm{N}_{i}}, t\right)+k_{i}\left(x_{j \in \mathrm{N}_{i}}, t\right) \cdot\left(\nabla\left\|x_{i}-z_{i}\right\|_{1}-w_{i}\right) .
\end{aligned}
$$

Now, the switching control term in $u_{i}(t)$, originally in (7), has been replaced by the continuous signal $w_{i}(t)$ which is obtained by (22) as the output of a low pass first-order filter fed by $\nabla\left\|x_{i}-z_{i}\right\|_{1}$. Besides, the dynamics of the auxiliary variables $z_{i}(t)$ has been reformulated as well as compared to (7). The reason will be more clear later on.

A proper choice for the time-constant $\tau_{i}$ of the filter is a trade-off between two conflicting requirements. Indeed, a too small value of $\tau_{i}$ yields a filter output still affected by high frequency components, thereby still causing chattering in the agent dynamics. On the other hand, a too large value of $\tau_{i}$ yields a decrease in the accuracy (namely, the agent's trajectories will deviate too much from the ideal ones that were achieved with the discontinuous local interaction rule).

The adopted method offers the advantage, over the alternative anti-chattering approaches that it still provides for the appearance of an ideal sliding motion along the manifold $x_{i}(t)-z_{i}(t)=0$, thereby making its achieved performance more predictable. We do not however claim that this method is superior to the others, as the actual performance may vary case by case and depending on the actual form of the uncertainty to be rejected. Every anti-chattering method, on the contrary, presents a trade-off, to be properly achieved case by case, between the removal of the high-frequency components from the plant control and the resulting, unavoidable, decrease in terms of accuracy and robustness.

Here, taking into account the closed loop dynamics (4), (21)-(23), by straightforward manipulations one gets also in the present case the discontinuous differential equation (14) and the corresponding differential inclusion (15). By analogous considerations, therefore, the trajectories of (4), (22) are constrained onto the invariant set $\boldsymbol{\sigma}(t)=\boldsymbol{\sigma}(t)=\mathbf{0}$ a.e. $\forall t \in \mathbb{R}_{\geqslant 0}$. Thus, the sliding motion generated by the control protocol (21)-(23) is the same as that in the original control (7).

Follows that the proposed anti-chattering reformulation provides stronger and more predictable robustness properties then, for instance [34] and [37], where no sliding motion can be established, since the discontinuous terms are replaced by some continuous approximations [23]. By inspection of (14) and (23) it is evident that the sliding motion is established on the dynamics of the auxiliary control variable $z_{i}(t)$ in (23), instead of in the agent dynamics (4), as the case of the protocol (7).

However, due to the different dynamics (23) of the auxiliary variable $z_{i}$ with respect to (7), agents trajectories will be different. By means of lengthy computations, taking advantage

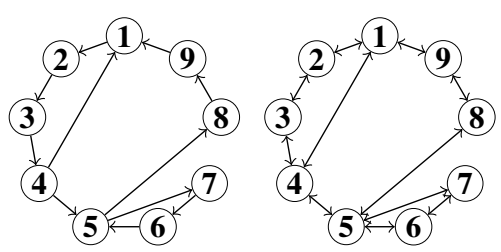

Fig. 1. Graph topologies for numerical simulations on, respectively, directed for Case 1 (left-plot), and undirected for Case 2 (right-plot).

of the treatment presented in [23], [37], one derives that the agents trajectories obtained using the anti-chattering local interaction rule (21)-(23) differ from the ideal ones, achieved using the discontinuous local interaction rule (7) by an $\mathcal{O}\left(\tau_{i}\right)$ mismatch. This result should not be surprising, indeed by substituting $\tau_{i}=0$ into (21)-(23) one recovers (7).

\section{NUMERICAL CASE STUDIES}

Two popular distributed control problems, referred as Case 1 and Case 2, are taken into account to validate the proposed distributed control strategies. In Case 1, the proposed scheme (7) is applied to robustify the traditional average consensus protocol for the distributed estimation of the average value of the agents' initial state over a weight-balanced directed graph. In Case 2 the strategy (7) is instead applied to robustify a MAS designed to solve a distributed optimization problem. Finally, Case 3 considers the chattering alleviation strategy (21)-(23) applied to the average consensus test Case 1, and the resulting performance is compared with that achieved by means of (7).

Simulation set-up: Simulations were performed on the MATLAB ${ }^{\circledR} /$ Simulink environment with the Euler fixed-step solver and sampling time $T_{s}=10^{-5}$ seconds. In both the test cases, an uncertain MAS consisting of $n=9$ agents is considered. The communication topologies for the tests are time-invariant and depicted in Fig. 1, resp., see the left-plot for Case 1 and the right-plot for Case 2. Each agent has two state variables $(d=2)$ with initial conditions

$$
x_{i}(0)=\left(x_{i, 1}(0), x_{i, 2}(0)\right)^{\top}=(i,-i)^{\top}, \quad \forall i=1,2, \ldots, 9 .
$$

The uncertain drift term $m_{i}\left(x_{j} \in \mathrm{N}_{i}, t\right)$ in (4) takes the form

$$
m_{i}\left(x_{j \in \mathrm{N}_{i}}, t\right)=\bar{m}_{i}\left(x_{i}\right)+\tilde{m}_{i}\left(x_{j \in \mathrm{N}_{i}}, t\right),
$$

where the known term is given by

$$
\bar{m}_{i}\left(x_{i}\right)=\left(\begin{array}{cc}
-1 & 0.1 \\
0.1 & -1
\end{array}\right) x_{i}
$$

and whereas the unknown term is as follows

$$
\tilde{m}_{i}(\cdot, t)=\overbrace{\tilde{\boldsymbol{\beta}}_{i} \cdot \operatorname{sign}\left(\sin \left(\tilde{\gamma}_{i} \cdot t\right)\right)}^{a_{i}(t)}+\overbrace{\sum_{j \in \mathrm{N}_{i}} \tilde{\epsilon}_{i j} \cdot \sin \left(\left(x_{i}-x_{j}\right) \cdot t\right)}^{b_{i}(x, t)}+\overbrace{\tilde{\boldsymbol{\alpha}}_{i} \cdot x_{i}}^{c_{i}(x)},
$$

where $\tilde{\boldsymbol{\alpha}}_{i} \in \mathbb{R}^{2 \times 2}, \tilde{\boldsymbol{\beta}}_{i} \in \mathbb{R}, \tilde{\boldsymbol{\gamma}}_{i} \in \mathbb{R}^{2}$, and $\tilde{\boldsymbol{\epsilon}}_{i j} \in \mathbb{R}$ are randomly chosen uncertain parameters selected within the following ranges: $\tilde{\boldsymbol{\alpha}}_{i}^{\ell j} \in[-0.2,0.2], \tilde{\boldsymbol{\beta}}_{i} \in[0,5], \tilde{\boldsymbol{\gamma}}_{i}^{\ell} \in[3,30], \tilde{\boldsymbol{\epsilon}}_{i j} \in[-1,0]$, $\forall i, j=1,2, \ell=1,2$. In accordance with (25), $a_{i}(t)$ denotes exogenous time-dependent perturbations, $b_{i}\left(x_{j \in \mathrm{N}_{i}}, t\right)$ represents the uncertain time-varying physical couplings among 
neighboring agents, and $c_{i}(x)$ models local uncertainties on the agent dynamics. A worst-case conservative upper-estimation of the uncertain term is made as follows $\left\|\tilde{m}_{i}(\cdot, t)\right\|_{\infty} \leq \delta_{i}(\cdot, t)=$ $6+0.2 \cdot\left\|x_{i}(t)\right\|_{\infty}$, on the basis of which the local gain $k_{i}(\cdot, t)$, to be used in (7) and (21)-(23), must satisfy $k_{i}(\cdot, t)>6+0.2$. $\left\|x_{i}(t)\right\|_{\infty}$. Here, we intentionally set $k_{i}(\cdot, t)=10+\left\|x_{i}(t)\right\|_{\infty}$, thus greater than necessary to shown some interesting features of the proposed chattering alleviation reformulation (21)-(23) of the nonsmooth control method (7).

Case 1 (Robust average consensus): Consider the widely known linear average consensus algorithm [3], implemented over a directed weight-balanced and strongly connected graph as that in the left-plot of Fig. 1, with edge weights $a_{12}=a_{23}=$ $a_{34}=2$ and $a_{i j}=1$ otherwise with $(i, j) \in \mathrm{E}$. The reference dynamics (5) for the uncertain MAS (4), (25) is as

$$
\dot{\hat{x}}_{i}=f_{i}\left(\hat{x}_{j \in \mathrm{N}_{i}}, t\right), \quad \hat{x}_{i}(0)=x_{i}(0), \quad \forall i \in \mathrm{V},
$$

with the reference local interaction rule chosen as follows

$$
f_{i}\left(\hat{x}_{j \in \mathrm{N}_{i}}, t\right)=-\sum_{j \in \mathrm{N}_{i}} a_{i j}\left(\hat{x}_{i}(t)-\hat{x}_{j}(t)\right) .
$$

The top-left pair of plots in Fig. 2 show the trajectories of the desired unperturbed MAS (26)-(27). The steady-state consensus value is

$$
\left(x_{1}^{*}, x_{2}^{*}\right)^{\top}=\frac{1}{9} \cdot \sum_{i=1}^{9}\left(x_{i, 1}(0), x_{i, 2}(0)\right)^{\top}=(5,-5)^{\top}, \quad \forall i \in \mathrm{V} .
$$

To compare our proposed method with a naive robustification approach based simply on the cancellation of the known terms of the agents' dynamics through feedback, we consider the control law $u_{i}(t)=f_{i}\left(x_{j \in \mathrm{N}_{i}}, t\right)-\bar{m}\left(x_{i}\right)$, whose resulting state trajectories are depicted in the top-right pair of plots in Fig. 2. As expected, due to uncertainties and perturbations neither a consensus among states, nor the estimation of the expected quantity (28) are achieved. In the bottom of Fig. 2 the agents trajectories obtained using the proposed robustification algorithm (7), (27) are displayed. According to Theorem 3, agents display the nominal behaviour of the reference MAS (26), (27).

The right plot of Fig. 3 shows the error signal $e_{4,1}(t)=$ $x_{4,1}(t)-\hat{x}_{4,1}(t)$. It confirms that the state trajectory obtained in the unperturbed case and that obtained in the perturbed case using the proposed control (7) are almost coincident from the initial time on (apart from a small error which is due the finite switching frequency of the numerical implementation). The price paid for a such high accuracy, which size is an order of the sampling-time $T_{S}$, is that the control (7) is discontinuous, as displayed in the left plot of Fig. 6, thus high frequency chattering in the control loop may appear.

Case 2 (Robust distributed optimization): Let us now consider the convex optimization problem

$$
\begin{array}{cc}
\text { minimize } & \sum_{i \in \mathrm{V}} h_{i}\left(x_{i}\right) \\
\text { subject to } & x_{i}=x_{j}, \forall(i, j) \in \mathrm{E},
\end{array}
$$

where $x_{i} \in \mathbb{R}^{2}$ and functions $h_{i}(\cdot)$ are chosen as follows

$$
h_{i}\left(x_{i}\right)=\frac{1}{2} x_{i}^{\top} \boldsymbol{Q}_{i} x_{i}+\boldsymbol{P}_{i}^{\top} x_{i}+\boldsymbol{R}_{i} \cdot e^{\mathbf{1}^{\top} x_{i}}, \quad i \in \mathrm{V}
$$
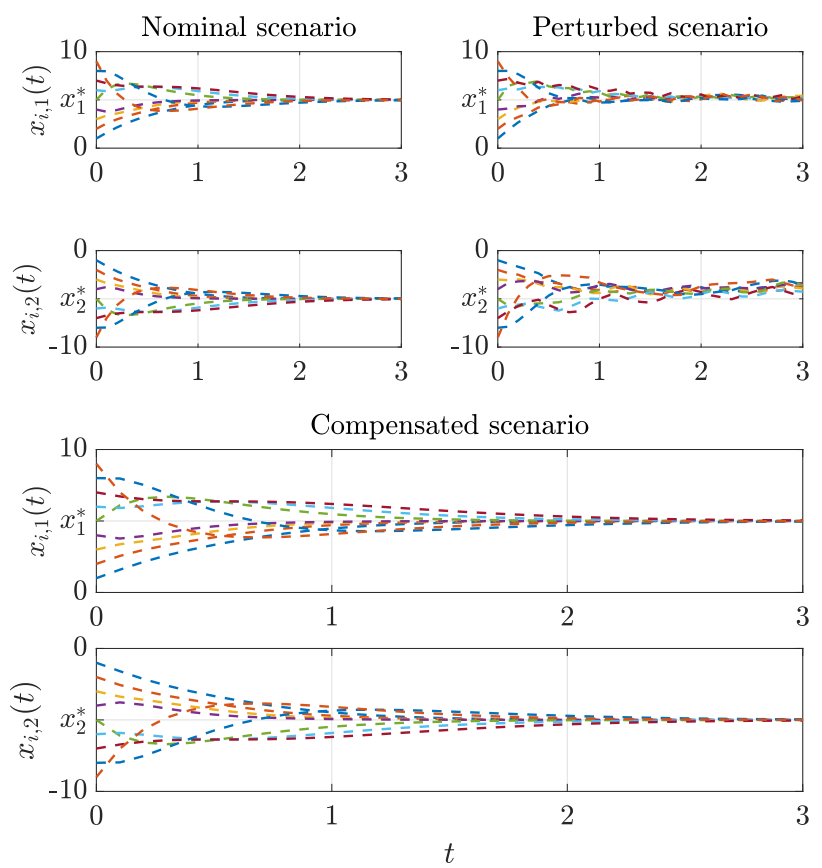

Fig. 2. Results on Case 1. Top-left: Agents trajectories in the perturbation-free scenario (26)- (27). Top-right: Agents trajectories in the perturbed scenario (4) under the control law $u_{i}=f_{i}-\bar{m}_{i}$. Bottom: Agents' trajectories in the perturbed scenario (4) under the robust local control (7), (27).
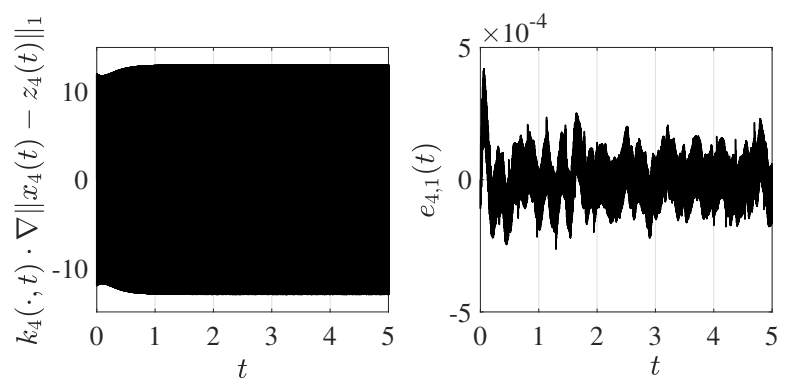

Fig. 3. Results on Case 1. Comparison between the evolution of the robustification control term $k_{4} \cdot \nabla\left\|x_{4}(t)-z_{4}(t)\right\|$ of the proposed nonsmooth control method (7), and the resulting steady accuracy $e_{4,1}(t)=x_{4,1}(t)-\hat{x}_{4,1}(t)$ for the "Case 1: Robust average consensus" discussed in Section V.

with $\mathbf{1}=(1,1)^{\top}$, and, $\boldsymbol{R}_{1}=0.5, \boldsymbol{R}_{2}=0.8, \boldsymbol{R}_{3}=0.9$, and,

$$
\begin{gathered}
\boldsymbol{Q}_{1}=\left(\begin{array}{ll}
0.2 & 0.1 \\
0.1 & 0.2
\end{array}\right), \boldsymbol{Q}_{2}=\left(\begin{array}{cc}
0.4 & 0.2 \\
0.2 & 3
\end{array}\right), \boldsymbol{Q}_{3}=\left(\begin{array}{cc}
1 & 0.2 \\
0.2 & 0.2
\end{array}\right) \\
\boldsymbol{P}_{1}=(-10,10)^{\top}, \boldsymbol{P}_{2}=(-20,20)^{\top}, \boldsymbol{P}_{3}=(-30,30)^{\top}
\end{gathered}
$$

whereas $\boldsymbol{R}_{4}=2 \boldsymbol{R}_{1}, \boldsymbol{R}_{5}=2 \boldsymbol{R}_{2}, \boldsymbol{R}_{6}=2 \boldsymbol{R}_{3}, \boldsymbol{R}_{7}=3 \boldsymbol{R}_{1}, \boldsymbol{R}_{8}=$ $3 R_{2}, R_{9}=3 R_{3}$, and, $Q_{4}=2 Q_{1}, Q_{5}=2 Q_{2}, Q_{6}=2 Q_{3}, Q_{7}=$ $3 Q_{1}, Q_{8}=3 Q_{2}, Q_{9}=3 Q_{3}$, and, $P_{4}=2 P_{1}, P_{5}=2 P_{2}, P_{6}=$ $2 \boldsymbol{P}_{3}, \boldsymbol{P}_{7}=3 \boldsymbol{P}_{1}, \boldsymbol{P}_{8}=3 \boldsymbol{P}_{2}, \boldsymbol{P}_{9}=3 \boldsymbol{P}_{3}$. The solution of the nonlinear convex programming problem (29), (30) is

$$
\left(x_{1}^{*}, x_{2}^{*}\right)^{\top}=(3.27,-3.02)^{\top} .
$$

Assuming the cost functions (31) to be private, thus available only to the agent $i$, we now aim to solve problem (29), (30) by means of a distributed algorithm which abides 

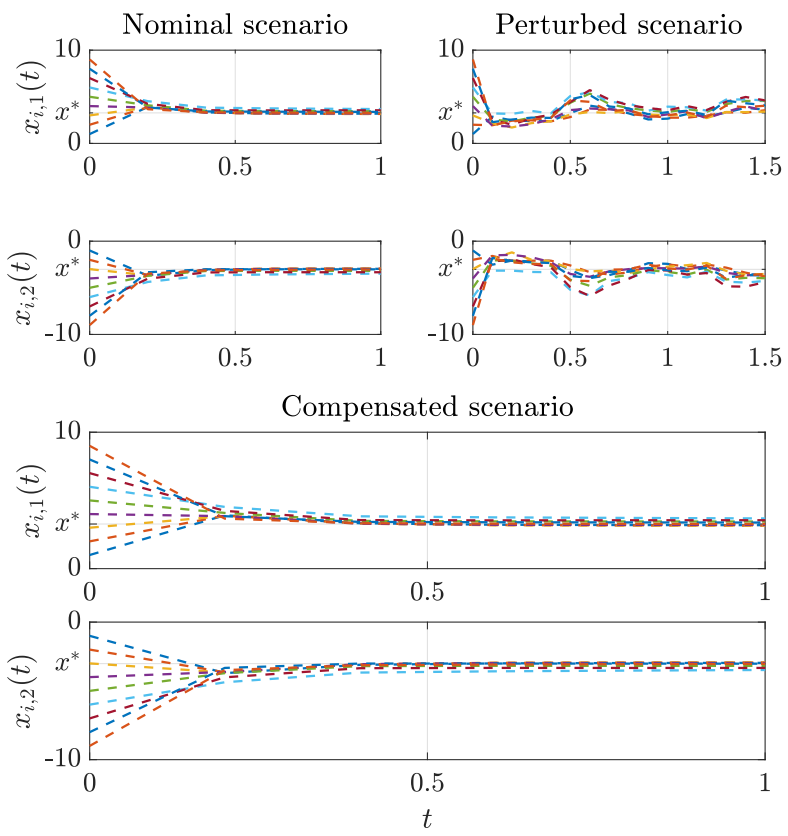

Fig. 4. Results on Case 2. Top-left: Agents' evolution in the perturbation free scenario (26), (33). Top-right: Agents' evolution in the perturbed scenario (4) under the control law $u_{i}=f_{i}-\bar{m}_{i}$. Bottom: Agents' evolution in the perturbed scenario under the robustifying control (7), (33).

the communication constraints represented by the undirected graph in the right-plot of Fig. 1 with unitary edge weights, $a_{i j}=1 \forall(i, j) \in \mathrm{E}$. To solve the proposed problem, we design the reference local interaction rule (or state update) as proposed in [9], [7], i.e., by adding the co-state additional control variables $v_{i} \in \mathbb{R}^{2}$ to each agent, representing Lagrange multipliers. Thus, the graph $G$ is connected and undirected, the MAS (5) converges to the minimizers (32) by means of the following reference local interaction rule (see [9], [7]),

$$
\dot{\hat{x}}_{i}=\left[\begin{array}{c}
\dot{x}_{i} \\
\dot{v}_{i}
\end{array}\right]=f_{i}\left(\hat{x}_{j \in \mathrm{N}_{i}}\right)
$$

where

$$
f_{i}(\cdot)=\left[\begin{array}{l}
-\mu_{1} \cdot \nabla h_{i}\left(x_{i}\right)+\sum_{j \in \mathrm{N}_{i}}\left(x_{j}-x_{i}\right)+\sum_{j \in \mathrm{N}_{i}}\left(v_{j}-v_{i}\right) \\
-\mu_{2} \cdot \sum_{j \in \mathrm{N}_{i}}\left(x_{i}-x_{j}\right)
\end{array}\right],
$$

with $\mu_{1}=50, \mu_{2}=100$. Furthermore, (25) changes as follows to account for the additional control variables $v_{i}$ in the reference local interaction rule

$$
m_{i}\left(x_{j \in N_{i}}, t\right)=\left[\begin{array}{c}
\bar{m}_{i}\left(x_{i}\right)+\tilde{m}_{i}\left(x_{j \in \mathrm{N}_{i}}, t\right) \\
0 \\
0
\end{array}\right] .
$$

Similarly to the results shown in Fig. 2 for Case 1, the top-left pair of plots of Fig. 4 shows the agents trajectories of the nominal reference MAS. The top-right pair of plots of Fig. 4 shows the trajectories obtained in the presence of the uncertain perturbations using a naive robustification approach based simply on the cancellation of the known terms of the agents dynamics through feedback, we consider the control law $u_{i}(t)=f_{i}\left(x_{i \in \mathrm{N}_{i}}, t\right)-\bar{m}_{i}\left(x_{i}\right)$, which does not converge any

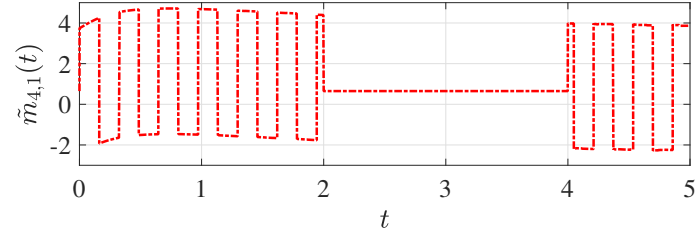

Fig. 5. Results on Case 3. Actual behaviour of the unknown uncertain dynamics $\tilde{m}_{4}(\cdot, t)=m_{4}(\cdot, t)-\bar{m}_{4}(\cdot, t)$. There, the time-varying uncertain terms $a_{4}$ and $b_{4}$ of $(25)$ has been intentionally set to zero within the time interval $t \in[2,4)$.
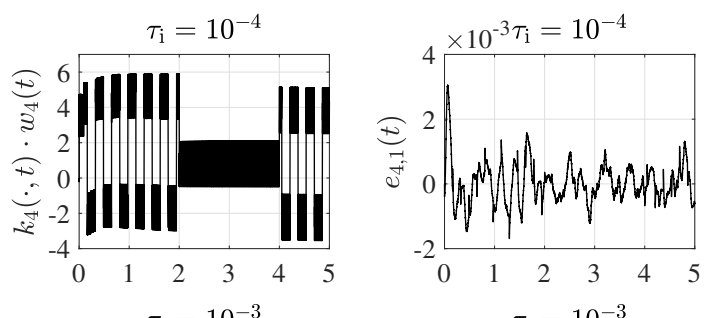

$\tau_{\mathrm{i}}=10^{-3}$
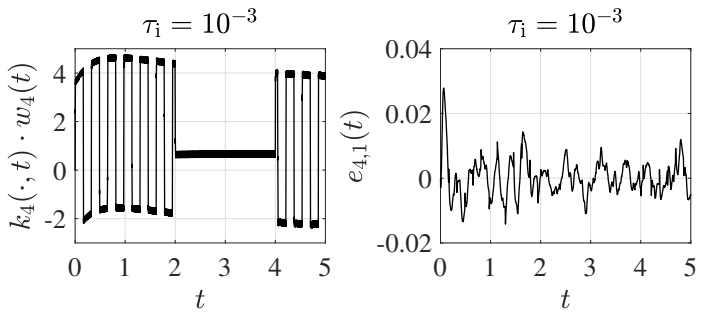

Fig. 6. Results on Case 3. Evolution of the robustification control term $w_{4,1}(t)$ of the chattering alleviation reformulation (21)-(23) of the proposed nonsmooth control (7) with respect to the unknown local uncertain term $\tilde{m}_{4}(\cdot, t)$ (left-column), and the resulting steady accuracy $e_{4,1}(t)=x_{4,1}(t)-\hat{x}_{4,1}(t)$ (right-column) for two values of filter's time-constant $\tau_{i}$. Both tests refers to the "Case 1: Robust average consensus" test case.

longer to the minimizers of (29), whereas the bottom plots of the same figure show the trajectories obtained in the presence of the uncertain perturbations using the proposed robustified protocol (7). It can be seen that the trajectories coincide with those of the reference unperturbed case thus restoring the correct convergence to the minimizer $x^{*}$ in (32), which solves the considered optimization problem (29)-(30).

Case 3 (Chattering alleviation): Here the chattering alleviation strategy (21)-(23) is tested and the corresponding performance is compared with that achieved using the original formulation of the proposed strategy provided in (7). This analysis refers to the average consensus test in Case 1. Tests with two different values of the filter time-constant $\tau_{i}$, which represents the unique additional tuning parameter associated to the smoothed version of the control, are provided. The results are shown in Fig. 6 for the fourth agent. The test labeled by $\tau_{i}=10^{-4}$ and $\tau_{i}=10^{-3}$ refer to two different realizations of the chattering alleviation reformulation (21)-(23).

During these tests, and accordingly with Fig. 5, we have artificially set to zero the uncertain terms $a_{4}(t)$ and $b_{4}(\cdot, t)$ of the fourth agent in (25) within the time interval $t \in[2,4)$. This has been made to verify that, using the chattering alleviation strategy, the control magnitude becomes smaller when the actual uncertainties and perturbations become smaller as well. 
The results of the comparative analysis are reported in Fig. 6. The plots in the two lines contains the results obtained with $\tau_{i}=10^{-4}$ and $\tau_{i}=10^{-3}$, whereas the first and second columns contain, respectively, the comparison of the robustification control $k_{4}(\cdot, t) \cdot w_{4,1}(t)$ of the chattering alleviation control (21)-(23) with respect to the unknown local uncertain term $\tilde{m}_{4}(\cdot, t)$, and the state error $e_{4,1}=x_{4,1}-\hat{x}_{4,1}$ between the actual and nominal reference trajectory.

From the plots in the left-column of Fig. 6 it is evident that as $\tau_{i}$ grows the actual control term " $k_{4}(\cdot, t) w_{4,1}(t)$ " becomes smoother and smoother. It is also seen that, for $t \in[2,4)$, where the uncertainties' magnitude is smaller, the magnitude of the control also reduces in accordance with the time evolution of the uncertainties to be rejected. This, instead, was not the case when the original protocol (7) was adopted. There, the control always switch between the extreme values of the convex set associated to the discontinuous control term, thus actuating a larger then necessary control effort, cf. Fig. 6 with Fig. 3 . The plots in the right column further shows that the accuracy $e_{4,1}(t)$ linearly depends on $\tau_{i}$, as stated in Section IV.

\section{CONCLUSIONS}

A robust protocol design process, based on the integral sliding mode control paradigm, devoted to provide robustness to a MAS affected by perturbations and uncertain agent dynamics executing given reference local interaction rules designed for agents modeled as $d$-dimensional continuous-time integrators, is presented. The method preserves the communication constraints between agents specified by the graph, and makes the trajectories of the uncertain MAS track exactly those of a reference MAS tasked to achieve a given coordination task in the nominal case. Future work will tackle the application of the proposed method to problems related to the control of smart grids. The same "robustification" task could be investigated from an input-output perspective, thereby considering agents with dynamics with relative degree greater than one or by relaxing the agents state availability assumption.

\section{REFERENCES}

[1] A. Pilloni, A. Pisano, and E. Usai, "Robust finite-time frequency and voltage restoration of inverter-based microgrids via sliding-mode cooperative control," IEEE Trans. Ind. Electron., 65(1), 907-917, 2018.

[2] J. W. Simpson-Porco, Q. Shafiee, F. Dörfler, J. C. Vasquez, J. M. Guerrero, and F. Bullo, "Secondary frequency and voltage control of islanded microgrids via distributed averaging," IEEE Trans. Ind. Electron., 61(11), 7025-7038, 2015.

[3] R. Olfati-Saber, J. Fax, and R. Murray, "Consensus and cooperation in networked multi-agent systems," Proc. IEEE, 95(1), 215-233, 2007.

[4] M. Ajina, D. Tabatabai, and C. Nowzari, "Asynchronous distributed event-triggered coordination for multiagent coverage control," IEEE Trans. Cybern., 2020.

[5] D. Bauso, L. Giarré, and R. Pesenti, "Non-linear protocols for optimal distributed consensus in networks of dynamic agents," Syst. Control Lett., 55(11), 918-928, 2006.

[6] J. Cortés, "Distributed algorithms for reaching consensus on general functions," Automatica, 44(3), 726-737, 2008.

[7] J. Wang and N. Elia, "Control approach to distributed optimization," IEEE Conf. Commun., Control, and Comput., 557-561, 2010.

[8] M. Franceschelli, A. Giua, and A. Pisano, "Finite-time consensus on the median value with robustness properties," IEEE Trans. Autom. Control, 62(4), pp. 1652-1667, 2017.

[9] B. Gharesifard and J. Cortés, "Distributed continuous-time convex optimization on weight-balanced digraphs," IEEE Trans. Autom. Control, 59(3), 781-786, 2014
[10] X. Wang, Y. Hong, and P. Yi, "Distributed optimization of multi-agent systems with unknown frequency disturbances," IEEE Chinese Control Conf., 1(1), 1772-1777, 2014.

[11] A. Garulli and A. Giannitrapani, "Analysis of consensus protocols with bounded measurement errors," Syst. Control Lett., 60(1), 44-52, 2011.

[12] D. Bauso, L. Giarré, and R. Pesenti, "Consensus for networks with unknown but bounded disturbances," Siam, J. on Control and Optim., 48(3), 1756-1770, 2009.

[13] M. Franceschelli, A. Pisano, A. Giua, and E. Usai, "Finite-time consensus with disturbance rejection by discontinuous local interactions in directed graphs," IEEE Trans. Autom. Control, 60(4), 1133-1138, 2015.

[14] M. Franceschelli, A. Pilloni, A. Pisano, A. Giua, and E. Usai, "Finitetime consensus with disturbance attenuation for directed switching network topologies by discontinuous local interactions," IEEE Conf. Decis. Control, 1(1), 2611-2616, 2013.

[15] A. Pilloni, A. Pisano, Y. Orlov, and E. Usai, "Consensus-based control for a network of diffusion pdes with boundary local interaction," IEEE Trans. Autom. Control, 61(9), 2708-2713, 2016.

[16] A. Pilloni, A. Pisano, M. Franceschelli, and E. Usai, "Finite-time consensus for a network of perturbed double integrators by second-order sliding mode technique," IEEE Conf. Decis. Control, 2145-2150, 2013.

[17] K. Yang, J. Huang, Y. Wu, X. Wang, and M. Chiang, "Distributed robust optimization (dro), part i: framework and example," Springer, Optimization and Eng., 15(1), 35-67, 2014.

[18] Y. Cao and W. Ren, "Finite-time consensus for multi-agent networks with unknown inherent nonlinear dynamics," Automatica, 50(10), 26482656, 2014.

[19] D. Xu, X. Wang, Y. Hong, and Z.-P. Jiang, "Global robust distributed output consensus of multi-agent nonlinear systems: An internal model approach," Syst. Control Lett., 87(1), 64-69, 2016.

[20] X. Wang, P. Yi, and Y. Hong, "Dynamic optimization for multiagent systems with external disturbances," Springer, Control Theory and Technol., 12(2), 132-138, 2014.

[21] Y. Jiang, J. Liu, and S. Wang, "Robust integral sliding-mode consensus tracking for multi-agent systems with time-varying delay," Asian J. of Control, 2014

[22] S. Yu and X. Long, "Finite-time consensus for second-order multi-agent systems with disturbances by integral sliding mode," Automatica, 54(1), 158-165, 2015.

[23] V. Utkin and J. Shi, "Integral sliding mode in systems operating under uncertainty conditions," IEEE Conf. Decis. Control, 4(1), 4591-4596, 1996.

[24] L. Fridman, A. Poznyak, F. J. Bejarano et al., Robust output LQ optimal control via integral sliding modes. Springer, 2016.

[25] A. Filippov, "Differential equations with discontinuous right-hand sides," Kluwer Academic Pub., London, 1988.

[26] B. Paden and S. Sastry, "A calculus for computing filippov's differential inclusion with application to the variable structure control of robot manipulators," IEEE Trans. Circuits Syst., 34(1), 73-82, 1987.

[27] D. Shevitz and B. Paden, "Lyapunov stability theory of nonsmooth systems," IEEE Trans. Autom. Control, 39(9), 1910-1914, 1994.

[28] A. Pilloni, A. Pisano, M. Franceschelli, and E. Usai, "Integral sliding modes for the robustification of consensus-based multi-agent based systems," IEEE Int. Workshop on Var. Structure Syst., 222-227, 2016.

[29] F. Clarke, "Optimization and nonsmooth analysis," Siam, 1990.

[30] F. H. Clarke, Y. S. Ledyaev, R. J. Stern, and P. R. Wolenski, "Nonsmooth analysis and control theory," Springer-Verlag New York, Inc., 1998.

[31] J. W. Simpson-Porco, F. Dörfler, and F. Bullo, "Synchronization and power sharing for droop-controlled inverters in islanded microgrids," Elsevier, Automatica, 49(9), 2603-2611, 2013.

[32] D. Bauso, "Consensus via multi-population robust mean-field games," Elsevier, Systems \& Control Letters, 107, 76-83, 2017.

[33] A. Pisano and E. Usai, "Sliding mode control: A survey with applications in math," Elsevier, Math. and Comput. in Simul., 81(5),954-979, 2011.

[34] J.-J. E. Slotine, "Sliding controller design for non-linear systems," Taylor \& Francis, Int. J. of control, 40(2), 421-434, 1984.

[35] F. Plestan, Y. Shtessel, V. Bregeault, and A. Poznyak, "New methodologies for adaptive sliding mode control," Taylor \& Francis, Int. J. of control, 83(9), 1907-1919, 2010.

[36] H. Lee and V. I. Utkin, "Chattering suppression methods in sliding mode control systems," Elsevier, Ann. Rev. in Control, 31(2), 179-188, 2007.

[37] V. I. Utkin, "Sliding modes in control and optimization," Springer Science \& Business Media, 2013. 